To appear in The Astrophysical Journal (1999 November 10 issue)

\title{
HOT MOLECULAR CORES AND THE FORMATION OF MASSIVE STARS
}

\author{
Mayra Osorio, Susana Lizano \\ Instituto de Astronomía, Unidad Morelia, UNAM, J.J Tablada 1006, \\ Col. Lomas de Santa María, 58090 Morelia, Michoacán, MEXICO \\ and \\ Paola D'Alessio \\ Instituto de Astronomía, UNAM, Apdo Postal 70-264, Cd. Universitaria, \\ 04510 México D. F., MEXICO
}

\begin{abstract}
It has been proposed that some hot molecular cores (HMCs) harbor a young embedded massive star, which heats an infalling envelope and accretes mass at a rate high enough to "choke off" an incipient HII region. This class of HMCs would mark the youngest phase known of massive star formation. In order to test this hypothesis, we model this type of object calculating the radiative transfer through a spherically symmetric dusty envelope infalling onto a central OB star, with accretion rates from $\dot{M}=6 \times 10^{-4}$ to $10^{-3} \mathrm{M}_{\odot} \mathrm{yr}^{-1}$. The dust thermal spectrum from infrared to radio wavelengths is derived and is compared with the observed fluxes of several hot cores which may be internally heated. We find that the data are best fitted using an envelope with the density distribution resulting from the collapse of a singular logatropic sphere, instead of that of a singular isothermal sphere. We conclude that several of these sources may be undergoing an intense accretion phase and find in all the cases that the accretion luminosity exceeds the stellar luminosity. We discuss the implications of this phase on the formation of massive stars.
\end{abstract}

Subject headings: stars: formation — dust - HII regions - ISM: individual (G34.24 $+0.13 \mathrm{MM}, \mathrm{W} 3\left(\mathrm{H}_{2} \mathrm{O}\right)$, Orion hot core, IRAS $\left.23385+6053\right)$ - radiative transfer

\section{INTRODUCTION}

One of the most interesting results of the past few years in the study of massive star formation is the discovery of Hot Molecular Cores (HMCs) which are small $(\lesssim 0.1 \mathrm{pc})$, dense $\left(n \sim 10^{6}\right.$ $\left.10^{8} \mathrm{~cm}^{-3}\right)$, hot $(\mathrm{T} \sim 100-300 \mathrm{~K})$ and dark $\left(A_{v} \sim 10^{3} \mathrm{mag}\right)$ molecular clumps. They are generally 
found in the proximity of ultra-compact HII (UCHII) regions, and are probably directly related to the formation of high mass stars. The best known example is the Orion-KL hot core (e.g., Genzel \& Stutzki 1989; Kaufman, Hollenbach \& Tielens 1998 and references therein) but several other cores have been observed with qualitatively similar characteristics: the core near G31.41+0.31 (Cesaroni et al. 1994b), G9.62+0.19F (Hofner et al. 1996), G34.24+0.13MM (Hunter et al. 1998, hereafter H98), W3 $\left(\mathrm{H}_{2} \mathrm{O}\right)$ (Turner \& Welch 1984), and IRAS 23385+6053 (Molinari et al. 1998; hereafter M98) among others. These sources have not been detected as free-free emitters at centimeter wavelengths; rather, their presence is established, and their physical properties determined through millimeter-wave and molecular line observations (see the review of Kurtz et al. 1999).

It is not yet understood what heats the gas in HMCs to a temperature higher than 100 $\mathrm{K}$. In some cases, the heating source might be a nearby star, facing the clump. This seems to be the case of the warm ammonia clumps associated with the source G61.48+0.09 (Gómez et al. 1995). Alternatively, Walmsley (1995) proposed that the heating source could be a recently formed OB-type star (or stars) inside the core undergoing an intense accretion phase. Following Yorke (1984), he suggested that high mass accretion rates of the infalling material could quench the development of an UCHII region, so that the free-free emission from the ionized material would be undetectable at centimeter wavelengths. Observationally, Cesaroni et al. (1994a) found that several HMCs seen in $\mathrm{NH}_{3}(4,4)$ are coincident with groups of water masers, which are known signposts of massive star formation. Thus, these sources are of particular interest because they may represent the youngest phase yet observed in the life of a massive star, and they could help us understand the process of high-mass star formation.

Kaufman et al. (1998) modeled the temperature distributions of internally and externally heated molecular cores and calculated the column density of hot dust and gas inside the cores. They considered constant density distributions and collapsing density distributions $\left(\rho \propto r^{-3 / 2}\right)$ for the cores. In the latter case, they did not discuss explicitly the effect of the accretion luminosity in the core heating. They applied their models to the Orion hot core with mass $M_{\text {core }} \sim 15 M_{\odot}$, and size $r_{\text {core }} \sim 0.01$ pc (i.e. a column density of $N\left(H_{2}\right)>5 \times 10^{24} \mathrm{~cm}^{-2}$ ). These authors concluded that an internal energy source is necessary to heat up the entire column density of the core to the observed temperatures, $T>200 \mathrm{~K}$, since the nearest known source (Radio Source "I") is not luminous enough to externally illuminate and produce the observed high temperatures of the gas and dust.

In this paper, we calculate the intrinsic dust thermal spectrum from infrared to radio wavelengths for a simple theoretical construct of the temperature profile of a HMC: a central massive star undergoing spherical accretion of a free falling envelope of gas and dust. The central heating source has two components, the stellar luminosity and the accretion luminosity.

We compare the spectrum of our model with the observed fluxes in several sources to determine the physical size of the HMC, the mass accretion rate of the envelope, and the spectral 
type of the central star. We present in $\S 2$ the parameters and the assumptions made in our model, in $\S 3$ the model results, in $\S 4$ a comparison with the available observations, in $\S 5$ a discussion our results, and in $\S 6$ the conclusions.

\section{THE MODEL}

Inspired by the scenario proposed by Walmsley (1995), we model a HMC as an envelope of gas and dust that is freely falling onto a recently formed massive central star. The young star is embedded within the dense core and interacts with it through its radiation, heating the core from inside.

We assume that the system consists of a central heating source, with a total bolometric luminosity $L_{\text {core }}$, surrounded by a spherically symmetric envelope, with inner radius $R_{\mathrm{d}}$ and outer radius $R_{\text {core }}$. The inner radius of the envelope is set by the dust destruction radius (see $\S 2.4$ ). The luminosity of the heating source, $L_{\text {core }}=L_{*}+L_{\text {acc }}$, has two components, the luminosity of the central star (considered to be a ZAMS massive star), $L_{*}$, and the accretion luminosity, $L_{\text {acc }}=G M_{*} \dot{M} / R_{*}$, where $G$ is the gravitational constant, $M_{*}$ is the stellar mass, $\dot{M}$ is the mass accretion rate, and $R_{*}$ is the stellar radius.

The effect of an energetic stellar wind is not taken into account. In spherical symmetry this is justified as long as the mass accretion rate is larger that the wind mass loss rate, $\dot{M}_{\text {acc }}>\dot{M}_{w}$, since the wind terminal speed is of the order of the free-fall velocity. In this case the ram pressure of the accretion flow will prevent the wind from escaping from the stellar surface. More likely, the angular momentum of the infalling envelope will deviate the flow from spherical accretion and the material will be deposited in a circumstellar disk around the star. In this case, the stellar winds could escape more easily through the poles. Our models will still be valid if the stellar winds are ejected in narrow bipolar cones, coexisting with the accreting envelope as in the case of low mass proto-stars (Adams, Lada \& Shu 1987), as long as these cones of missing material represent only a small fraction of the total core material and provided the axis of the bipolar outflow is not close to the line-of-sight. Also, the deviations of the envelope density profile from spherical symmetry will be important only within the centrifugal radius (Adams \& Shu 1986). This region does not make a significant contribution to the emergent flux as long as it is small compared to the size of the core. Since this is the case expected for our models, the predicted spectra will be appropriate.

To calculate the emergent dust thermal spectrum, we ignore the interaction of radiation from the central object with envelope matter located inside $R_{\mathrm{d}}$. We calculate the flux received from the source by an observer at a distance $D$ by integrating the equation of radiative transfer along rays for different impact parameters (e.g. Mihalas 1978). We take the volume emissivity of the dust to equal the LTE value $\rho \kappa_{\nu} B_{\nu}(T)$, where $\rho$ is the matter density, $\kappa_{\nu}$ is the monochromatic absorption opacity per unit mass, $B_{\nu}$ is the Planck function, and $T$ is the local dust temperature. In order to solve the transfer equation, a knowledge of $\rho, \kappa_{\nu}$ and $T$ is needed. 


\subsection{Density Distribution}

Two different types of density distributions are considered: one resulting from the collapse of the singular isothermal sphere (SIS; Shu 1977), and the other from the collapse of the singular logatropic sphere (SLS; McLaughlin \& Pudritz 1997). The logatropic equation of state, $P=P_{0} \ln \left(\rho / \rho_{0}\right)$, has been invoked to explain the linewidth-size relation observed in molecular clouds (Lizano \& Shu 1989; Myers \& Fuller 1992; McLaughlin \& Pudritz 1996) since the sound speed, $c_{s}$, given by $c_{s}^{2}=d P / d \rho=P_{0} / \rho$, behaves like the observed velocity dispersion in these clouds. In the above equations, $P_{0}$ has a constant value and $\rho_{0}$ is an arbitrary reference density.

The logatropic and isothermal collapses occur in the same general fashion: an expansion wave moves outward into a cloud at rest and the gas behind the wave falls into the central proto-star. The infalling matter close to the center has free-fall density and velocity profiles given

by $\rho=\dot{M}\left(32 \pi^{2} G M_{*}\right)^{-1 / 2} r^{-3 / 2}$, and $v=-\left(2 G M_{*}\right)^{1 / 2} r^{-1 / 2}$, respectively, while in the outer region the density profile tends towards the hydrostatic equilibrium configuration: $\rho(r)=a^{2}(2 \pi G)^{-1} r^{-2}$ for the SIS, where $a$ is the isothermal sound speed, and $\rho(r)=\left(P_{0} / 2 \pi G\right)^{1 / 2} r^{-1}$ for the SLS. Nevertheless, there are important differences in the behavior of both types of collapsing clouds. The mass accretion rate is constant for the SIS collapse: $\dot{M}^{i}=m_{0}^{i} a^{3} / G$, where the reduced mass is $m_{0}^{i}=0.975$. For the SLS collapse, however, the mass accretion rate is a steep function of time given by

$$
\dot{M}=\frac{\left(2 \pi G P_{0}\right)^{3 / 2}}{4 G} m_{0} t^{3}
$$

and the reduced mass is $m_{0}=0.0302$.

For a given stellar mass, $M_{*}$, and present mass accretion rate, $\dot{M}$, the age of the system in the SIS is given by $t_{\text {age }}^{i}=M_{*} / \dot{M}$, while it is longer for the SLS

$$
t_{\text {age }}=4 \frac{M_{*}}{\dot{M}}=4 t_{\text {age }}^{i}
$$

Furthermore, for a given central star, the mass of the infalling envelope is given by

$$
M_{\mathrm{env}}=M_{*}\left(\frac{m(x)}{m_{0}}-1\right),
$$

where $m(x)$ is the dimensionless mass function of the dimensionless variable $x$. For the SIS, $x=r / a t$, where $r$ is the distance to the central star, while $x=4\left(2 \pi G P_{0}\right)^{-1 / 2} r t^{-2}$, for the SLS. For $t_{\text {age }}$, the location of the expansion wave, $r_{\mathrm{ew}}$, occurs at $x=1$. Then, one has the infalling density distribution within $r_{\text {ew }}$ and the hydrostatic density distribution outside this radius. In particular, at the expansion wave, $m(1)=2$ for the SIS and $m(1)=1$ for the SLS. Thus, at a given time, within the expansion wave, the SLS has only $3 \%$ of the mass in the star and the rest is contained in the envelope. In contrast, for the SIS, $m_{0}^{i}=0.975$, so about half of the mass is in the star and half in the envelope. Also, eqns. (2) and (3) imply that, for a given $M_{*}$ and $\dot{M}$, the isothermal envelope has less mass than the logatropic envelope. For simplicity, we assume that the density distribution is not affected by effects of the finite core size. 


\subsection{Dust Opacity}

We assume that the dust in the core is a mixture of 4 types of grains: graphite, silicates, iron and water ice, with optical constants and abundances taken from D'Alessio (1996; see references therein), and a MRN-type size distribution (Mathis, Rumpl \& Nordsieck 1977). Water ice only contributes to the opacity for $\mathrm{T}<100 \mathrm{~K}$. For higher temperature we assume it has sublimated (Sandford \& Allamandola 1993). Since little is known about grain opacities in the sub mm range, we adopt a power law of the form, $\kappa_{\lambda} \propto \lambda^{-\beta}$, with $1 \leq \beta \leq 2$ for $\lambda \geq 200 \mu \mathrm{m}$. With these monochromatic opacities, the Rosseland mean opacity, $\chi_{\mathrm{R}}$, and the Planck mean opacity, $\kappa_{\mathrm{P}}$, are calculated in the wavelength range $0.1<(\lambda / \mu m)<10^{5}$. In this range, the monochromatic opacities for the low and high temperatures in the envelope $(\sim 20-2000 \mathrm{~K})$, are well represented.

\subsection{Temperature Profile of the Dust Envelope}

Kenyon, Calvet \& Hartmann (1993) made detailed models of the temperature structure and emission of dusty infalling envelopes around low mass proto-stars. They proposed a simplified calculation of the temperature profile which we have adopted to calculate the temperature distribution in our model. The basic idea of this approximate treatment considers radiative equilibrium for the outer optically thin dusty envelope and assumes the standard diffusion approximation in the inner optically thick region. The radius that divides the optically thin and thick regions is called the "photospheric radius", $R_{\mathrm{ph}}$. The total luminosity is conserved at this radius, thus, $L_{\text {core }}=4 \pi R_{\mathrm{ph}}^{2} \sigma T_{\mathrm{ph}}^{4}$, where $\sigma$ is Stefan-Boltzmann constant and $T_{\mathrm{ph}}=T\left(R_{\mathrm{ph}}\right)$. We also require that $\tau_{\mathrm{R}}\left(T_{\mathrm{ph}}\right)=2 / 3$, where $\tau_{\mathrm{R}}\left(T_{\mathrm{ph}}\right)$ is the Rosseland mean optical depth weighted by the Planck function evaluated at $T_{\mathrm{ph}}$. Both, the optical depth and the luminosity conservation conditions are used to determine $R_{\mathrm{ph}}$ and $T_{\mathrm{ph}}$. Unlike the SIS collapse density distribution, in the collapse of a SLS the photospheric radius changes for different external radii of the envelope because the external part of the SLS envelope has an appreciable optical depth.

The equation for radiative equilibrium is

$$
\int_{0}^{\infty} \kappa_{\nu} B_{\nu}[T(r)] d \nu=\int_{0}^{\infty} \kappa_{\nu} J_{\nu} d \nu
$$

For the optically thin outer region, the mean intensity, $J_{\nu}(r)$, is approximated as $J_{\nu}(r)=W(r) B_{\nu}\left(T_{\mathrm{ph}}\right)$, where $W(r)$ is the dilution factor: $W(r)=\frac{1}{2}\left(1-\left[1-\left(R_{\mathrm{ph}} / r\right)^{2}\right]^{\frac{1}{2}}\right)$. Thus, one accounts for the geometric dilution of the radiation field of the envelope's photosphere but not the exponential attenuation of this emission which, by assumption, is less than $\mathrm{e}^{-2 / 3}$. Therefore, we consider that the grains in this region are heated only by the energy from the photosphere and we neglect the heating by the diffuse field from the optically thin region. Using the Planck mean opacities, the thermal balance equation for the dust becomes

$$
T^{4} \kappa_{\mathrm{P}}(T)=W(r) T_{\mathrm{ph}}^{4} \kappa_{\mathrm{P}}\left(T_{\mathrm{ph}}\right) .
$$


where the Planck mean opacity, $\kappa_{\mathrm{P}}(T)$, is evaluated at the local dust temperature, $T$, and $\kappa_{\mathrm{P}}\left(T_{\mathrm{ph}}\right)$ is evaluated at $T_{\mathrm{ph}}$. Once the photospheric temperature is known, the implicit eqn. (5) determines the temperature distribution, $T(r)$ in the outer region.

The innermost regions of the accreting dust envelope (inside the photospheric radius) are optically thick. In such regions the temperature gradient is determined by the standard diffusion approximation

$$
L_{\mathrm{core}}=-\frac{64 \pi \sigma r^{2} T^{3}}{3 \chi_{\mathrm{R}} \rho} \frac{d T}{d r} .
$$

For a given density $\rho(r)$ and Rosseland mean opacity $\chi_{\mathrm{R}}(T)$, one solves this equation for the temperature distribution $T(r)$, using a Runge-Kutta integration technique subject to the boundary condition $T\left(R_{\mathrm{ph}}\right)=T_{\mathrm{ph}}$. The approximation used here to obtain the temperature distribution is a very simplified approach since a full angular and frequency-dependent radiative transfer treatment is beyond the scope of this paper.

\subsection{Dust Destruction Radius}

The dust is sublimated for temperatures higher than $1200 \mathrm{~K}$ (e.g. Adams \& Shu 1985), producing an inner dust free cavity. The dust destruction radius is calculated following the evolution of an average sized dust particle $\left(a_{g}=0.1 \mu \mathrm{m}\right)$ until it disappears, neglecting particle accretion onto the grains. The equation for the grain radius variation is given by

$$
\left(\frac{d a_{g}}{d t}\right)_{\mathrm{sub}}=\frac{-1}{\rho_{g}} \sqrt{\frac{3 \mu_{g} m_{H}}{16 k T(r)}} P_{\mathrm{vap}},
$$

where $\rho_{\mathrm{g}}$ is the grain mass density, $\mu_{\mathrm{g}}$ is the mean molecular weight of the gas and dust material, $m_{\mathrm{H}}$ is the hydrogen mass and $P_{\text {vap }}$ is the vapor pressure, taken from Lamy (1974). $P_{\text {vap }}$ depends on the dust grain composition, which we have assumed to be pure silicate. With this approximation one obtains $T\left(R_{\mathrm{d}}\right) \sim 1200 \mathrm{~K}$, where the dust destruction radius, $R_{\mathrm{d}}$, is of order tens of AUs (see Tables $1 \mathrm{a}, 1 \mathrm{~b}$ and 3 ). In a more rigorous analysis one should calculate the destruction radius of each one of the components and sizes of the dust grain mixture.

\subsection{Central Stars}

To determine the total luminosity, we must specify not only the stellar mass and mass accretion rate but also the radius and luminosity of the central star. It is unclear whether a star formed under very high accretion rates, will have a normal ZAMS radius. In fact, Adams \& Shu (1985), using the results of Stahler, Shu \& Taam (1980) for the formation of low mass stars, found that the stellar radius is an increasing function of accretion rate: $R_{*} \propto \dot{M}^{0.33}$. Furthermore, Stahler, Palla \& Salpeter (1986) studying the formation of primordial massive stars, found the 
same type of behavior: $R_{*} \propto \dot{M}^{0.41}$. In addition, Beech \& Mitalas (1994) and Bernasconi \& Maeder (1996) have studied the formation of massive stars with solar abundances and under constant accretion rates. These authors do not discuss enlarged radii, maybe because they studied only accretion rates $<10^{-4} M_{\odot} \mathrm{yr}^{-1}$. It is then quite probable that $\mathrm{OB}$ stars, formed under intense accretion flows inside HMCs, will have radii larger than the ZAMS values since they have not had time to get rid of excess internal energy. We consider two possibilities: stars with ZAMS radii of Thompson (1984) (see also Vacca, Garmany, \& Shull 1996), and stars with stellar radii $R_{*}=10^{12} \mathrm{~cm}$. This last value for the radius is a factor of $\sim 2-5$ larger than the ZAMS radii for the central B0-B3 stars considered in our models and is within the range of radii found by the authors mentioned above. Finally, we adopt the ZAMS luminosities of Thompson (1984; see also Vacca, Garmany, \& shull 1996), even though, the luminosities of massive stars forming under intense accretion rates are also uncertain.

\section{MODEL RESULTS}

This section presents the results obtained for a set of models with different values of the mass accretion rate, the external radius of the envelope, and the spectral type of a ZAMS central star. We discuss the effects in the spectrum, when the above parameters are changed, assuming the collapse density distribution of the SLS and SIS. For both cases, a distance of $4.9 \mathrm{kpc}$ was assumed for the model source, namely the distance to IRAS $23385+6053$, which is one of the studied HMCs.

The summary of the physical properties of the SLS models is given in Table 1a and its general trends are shown in Figure 1a. The top panel of Figure 1a shows the spectra obtained for three different values of the mass accretion rate $\left(\dot{M}=1.5 \times 10^{-3}, 4.4 \times 10^{-4}\right.$ and $\left.8.1 \times 10^{-5} M_{\odot} \mathrm{yr}^{-1}\right)$, for a B0 central star with a radius $R_{*}=4 \times 10^{11} \mathrm{~cm}$ (Thompson 1984), and an envelope with an external radius $R_{\text {core }}=0.1 \mathrm{pc}$. As seen in the figure, the peak flux density increases and shifts to longer wavelengths as the accretion rate increases. This behavior can be explained as a result of the increase of the optical depth in the envelope, because of the overall density increase for higher accretion rates. As a consequence of the increase in opacity, the flux tends to be redistributed towards longer wavelengths, where the envelope is optically thinner, producing a shift of the peak of the spectrum. This also has the effect of increasing the depth of the silicate absorption feature at $10 \mu \mathrm{m}$. The total luminosity, $L_{\text {core }}$, increases with the mass accretion rate because of the increase in the accretion luminosity. This results in higher values of the peak flux density. Table 1a shows that the photospheric radius increases and its temperature decreases with increasing mass accretion rates.Again this is easily understood as a result of the overall density increase in the envelope that moves the photospheric radius to outer (and thus cooler) regions, as the accretion rate increases. Table 1 a also indicates that the dust destruction radius, $R_{\mathrm{d}}$, increases with the accretion rate, since the central luminosity, $L_{\text {core }}$, increases.

The middle panel of Figure 1a shows the spectra for different spectral types of the central 
star (O7, B0 and B3), for an envelope with external radius of $0.1 \mathrm{pc}$, and an accretion rate of $4.4 \times 10^{-4} M_{\odot} \mathrm{yr}^{-1}$. The peak flux density of the spectrum shifts to longer wavelengths for later spectral types. This happens because, for the models presented in this figure, $R_{\text {core }}<r_{\text {ew }}$ (see column 7 in Table 1a), thus, the mass of the envelope is given by eqn. (3), where $m(x) \propto x^{1.84}$, and $x \propto M_{*}^{-1} \dot{M}^{2 / 3}$. Hence, the envelope mass is inversely proportional to the stellar mass, i.e. $M_{\text {env }} \propto M_{*}^{-0.84}$ (see last column in Table 1a). Therefore, the opacity of the envelope increases for later spectral types and the emission is redistributed towards longer wavelengths.

Finally, the bottom panel of Figure 1a illustrates the trends of the spectra for different external radii of the envelope $\left(R_{\text {core }}=0.15,0.10\right.$ and $\left.0.05 \mathrm{pc}\right)$, for a central star with spectral type $\mathrm{B} 0$ and an accretion rate of $4.4 \times 10^{-4} M_{\odot} \mathrm{yr}^{-1}$. As seen in the figure, the flux at long wavelengths increases slightly, while it decreases slightly at short wavelengths, as the external radius increases. This effect can be also understood as a consequence of the redistribution of the flux density to longer wavelengths, because of the increase of the optical depth for larger external radii. Note that, the photospheric radius increase with increasing external radii, as discussed in $\S 2.3$.

The summary of the physical properties of the SIS models is given in Table $1 \mathrm{~b}$ and its general trends are shown in Fig 1b. The SIS models in each panel were computed with the same parameters that the SLS models (see columns 1 and 2 in Tables 1a and 1b). As one can see from Figures 1a and 1b, that the general behavior of the SIS models in each panel is the same the SLS models. However, one can also see that the spectra resulting of the SIS models have smaller millimeter fluxes and greater infrared fluxes than the SLS. This happens because the mass of the SIS envelope, $\mathrm{M}_{\mathrm{env}}=\dot{M}\left(18 G M_{*}\right)^{-1 / 2} R_{\text {core }}^{3 / 2}$, is always smaller than that of the SLS envelope (see last columns in Tables 1a and 1b). Thus, the SIS envelope is optically thinner and emits most of the radiation at shorter wavelengths than the SLS envelopes.

In summary, the spectrum at all frequencies is very sensitive to the mass accretion rate; infrared flux densities are sensitive to the spectral type of the central star, and the value of the external radius has only a small effect on the spectrum for the parameters explored in Figures 1a and $1 b$.

\section{COMPARISON OF THE MODEL WITH OBSERVATIONS}

To date, about 20 candidate HMCs have been proposed (Kurtz et al. 1999). In general, these objects are associated with nearby or embedded FIR sources and/or UCHII regions. Thus, it is very likely that the observed fluxes are contaminated by these nearby sources and overestimate the actual contribution of the HMC, making it difficult to fit the intrinsic spectrum. For this reason, we selected relatively isolated objects, and among them, those with the most complete data sets available in order to constrain, as much as possible, the parameters of our model. With these restrictions, we chose the sources: G34.24+0.13MM, W3 $\left(\mathrm{H}_{2} \mathrm{O}\right)$, Orion hot core and IRAS $23385+6053$ as a representative sample to test the model. 
In order to match the sources, fits using both the SLS and SIS density distributions have been considered. Figure 2 shows the observed fluxes of the source G34.24+0.13MM (H98) and the results of three model spectra for HMCs with $R_{\text {core }}=0.07 \mathrm{pc}$ and a central B3 star with $10 M_{\odot}$. The dotted line corresponds to the SIS collapse density distribution with $\dot{M}=1.0 \times 10^{-3} M_{\odot} \mathrm{yr}^{-1}$, while the dot-dashed line corresponds to the SLS collapse density distribution with $\dot{M}=6.5 \times 10^{-4} M_{\odot} \mathrm{yr}^{-1}$. For both models we used a ZAMS stellar radius, $R_{*}=2.0 \times 10^{11} \mathrm{~cm}$ (Thompson 1984). In order to match the observed fluxes at mm wavelengths, massive envelopes with high accretion rates are required. In particular, for a given mass accretion rate, the envelope is less massive for the SIS than the SLS, so one requires a higher mass accretion rate, producing a flux density at $20 \mu \mathrm{m}$ several orders of magnitude higher than the upper limit on the flux at this wavelength (see discussion in $\S 3$ ). The logatropic collapse model, on the other hand, has a smaller excess emission at $20 \mu \mathrm{m}$ which can be attenuated invoking a high external extinction to the HMC, $A_{V} \sim 100$ mag. This value of the external extinction is somewhat high, even though HMCs are found inside dense regions in molecular clouds. More generally accepted values are $A_{V} \lesssim 30 \mathrm{mag}$ (e.g. Testi et al 1998).

The solid line in Figure 2 shows the spectrum of the SLS model discussed above, but for a star of radius $R_{*}=10^{12} \mathrm{~cm}$, as discussed in $\S 2.5$. Clearly, the excess of emission at $20 \mu \mathrm{m}$ is reduced, and now fits the constraint at $20 \mu \mathrm{m}$, with no external extinction required, since the accretion luminosity decreases for this large stellar radius. Note that if this large stellar radius were used for the SIS density distribution, one still requires $A_{V}>100$ mag to attenuate the excess emission at $20 \mu \mathrm{m}$.

A similar result is found for the remaining sources, so, for these, we present only models for a SLS collapse distribution. In addition, the central stars are assumed to have radii $R_{*}=10^{12}$ $\mathrm{cm}$, and any external extinction is neglected. It is likely that in reality, a combination of some external extinction $\left(A_{V} \lesssim 30 \mathrm{mag}\right)$ plus a star with a radius larger than its ZAMS value, could be responsible for the observed spectrum. Finally, similar trends, as those shown in Figure 1, are found for these type of central stars with large radii.

\subsection{G34.24 + 0.13MM}

The source G34.24 + 0.13MM discovered by Hunter et al. (1998), is located 84" (1.5 pc) southeast of the UCHII region G34.26 + 0.15, within the G34.3 + 0.2 HII region complex, which is at a distance of $3.7 \mathrm{kpc}$ (Kuchar \& Bania 1994). H98 identified G34.24 + 0.13 MM as a massive proto-stellar object, which coincides with a methanol maser. This source has upper limits on the centimeter continuum emission of $\mathrm{S}_{\nu} \lesssim 0.8 \mathrm{mJy}$ at $15 \mathrm{GHz}$, and $\mathrm{S}_{\nu} \lesssim 0.6 \mathrm{mJy}$ at $8.3 \mathrm{GHz}$ (Goss 1999, personal communication). From observations at $1.3 \mathrm{~mm}$ and $2.7 \mathrm{~mm}$, H98 measured an angular diameter of $2^{\prime \prime}(0.037 \mathrm{pc})$. They also obtained near-infrared images and photometry at 10 and $20 \mu \mathrm{m}$, but no infrared source was detected coincident with the millimeter position. H98 estimated a total luminosity in the range of $1600-6300 \mathrm{~L}_{\odot}$. These authors argue that because 
of the high luminosity and the lack of cm compact radio continuum emission the core probably contains a deeply embedded proto-B star, since there is no nearby source that could provide the observed heating.

We fitted the observed spectrum of this source with a model requiring a central B3 star, $\dot{M}=6.5 \times 10^{-4} M_{\odot} \mathrm{yr}^{-1}, R_{\text {core }}=0.07 \mathrm{pc}$, and an opacity index $\beta=2$ (see Table 2 ). Earlier spectral types than B3 are too luminous and are incompatible with the observed mid-infrared emission (see trends of the models in the middle-panel of Figure 1a). Accretion rates smaller than the value $\dot{M}=6.5 \times 10^{-4} M_{\odot} \mathrm{yr}^{-1}$ do not reproduce the observed millimeter emission (see the trends of the models in the top-panel of Figure 1a). The top-left panel of Figure 3 shows the spectrum. Table 3 lists the physical properties of the source derived with this model: the dust destruction front, $R_{\mathrm{d}}$; the photospheric radius, $R_{\mathrm{ph}}$; the photospheric temperature, $T_{\mathrm{ph}}$; the molecular volume density at the external radius, $n\left(R_{\text {core }}\right)$; the total luminosity, $L_{\text {core }}$; the fraction of the accretion luminosity, to the total luminosity, $L_{\text {acc }} / L_{\text {core }}$; the core mass within $R_{\text {core }}, M_{\text {core }}$; the total column density $N\left(H_{2}\right)$; the present age of the core given by eqn. (2); and the expansion wave radius.

The required central B3 star is late enough so that it will not develop an HII region detectable from $3.7 \mathrm{kpc}$. Even if the star were earlier than B3, one would not expect centimeter radio emission since the critical mass accretion rate that would quench the development of an UCHII region (see Walmsley 1995 , eqn. [1]) is only $\dot{M}_{\text {crit }}=3.20 \times 10^{-8} M_{\odot} \mathrm{yr}^{-1}$, considerably smaller than that implied by our model (see Table 2).

\section{2. $\mathrm{W} 3\left(\mathrm{H}_{2} \mathrm{O}\right)$}

About $6^{\prime \prime}(0.06 \mathrm{pc})$ east of the UCHII region $\mathrm{W} 3(\mathrm{OH})$ located at a distance of $2.2 \mathrm{kpc}$ (Humphreys 1978), a luminous embedded young star was discovered near a complex of $\mathrm{H}_{2} \mathrm{O}$ masers by Turner \& Welch (1984; hereafter the TW object or $\left.\mathrm{W} 3\left(\mathrm{H}_{2} \mathrm{O}\right)\right)$. This object is manifest by a warm, dense concentration of molecular material (Turner \& Welch 1984; Wilson, Gaume \& Johnston 1993; Wink et al. 1994), a bipolar outflow traced by VLBI proper motions of $\mathrm{H}_{2} \mathrm{O}$ maser spots (Alcolea et al. 1992) emanating from a small, elongated synchrotron emission source (Reid et al. 1995; Wilner, Reid \& Menten 1999). Additionally, Wilner, Welch \& Forster (1995), and Wyrowski et al. (1997) detected a compact $\left(\sim 2^{\prime \prime}=0.02 \mathrm{pc}\right) \mathrm{mm}$ continuum source, that was interpreted as thermal dust continuum emission from the $\mathrm{W} 3\left(\mathrm{H}_{2} \mathrm{O}\right)$ hot core. Recent molecular line and continuum interferometric observations at $1.4 \mathrm{~mm}$, reaching subarcsecond angular resolution (Wyrowski et al. 1999), indicate that the continuum emission from $\mathrm{W} 3\left(\mathrm{H}_{2} \mathrm{O}\right)$ splits into three components. The eastern component (component A in the nomenclature of Wyrowski et al. 1999) coincides with the source of the maser outflow and synchrotron emission, and is associated with a $200 \mathrm{~K}$ temperature peak, derived from molecular line transitions. Nevertheless, since our models are spherically symmetric, we model the spectrum of the compact source as observed by Wilner et al. (1995) and Wyrowski et al. (1997) with lower spatial resolution. 
We fit the spectrum of this source with a model requiring a central B0 star, $\dot{M}=1.2 \times 10^{-3} M_{\odot} \mathrm{yr}^{-1}, R_{\text {core }}=0.05 \mathrm{pc}$, and an opacity index $\beta=1.6$ (see Table 2 ). Table 3 lists the physical properties of this model and the resulting spectrum is shown in the upper right panel of Figure 3. Because of the few data points available for this source, the model fit was not unique, and we selected the model solution with the spectral type of the central star and the index of the opacity law in accordance with those inferred by Wyrowski et al. (1997, 1999). High angular resolution observations at mid- and far-infrared wavelengths are necessary to constrain the total luminosity of this source, and the spectral type of the central star.

\subsection{Orion hot core}

Because of its proximity ( $D=480 \mathrm{pc}$; Genzel et al. 1981), the most studied region of high-mass star formation is the Orion molecular cloud (OMC-1). Line studies of the central portion of OMC-1 (i.e., the Kleinmann-Low nebula) showed that it was separated into a number of velocity components that were subsequently associated with several spatial components as very high-resolution mapping became available (see Wright et al. 1996 and references therein). Prominent at the center of this region is a dense condensation of gas and dust known as the "Orion hot core" which has a gas density $n\left(\mathrm{H}_{2}\right) \gtrsim 10^{7} \mathrm{~cm}^{-3}$ and a temperature of $150-300 \mathrm{~K}$ (see Kaufman et al. 1998 for references). Whether this source is heated either externally (Blake et al. 1996, Wright, Plambeck \& Wilner 1996) or internally (Masson \& Mundy 1988) is still a matter of great debate. In addition, Chandler \& Wood (1997) suggested that there is an HII region associated with Radio Source "I", located to the northwest of the hot core ( 2 " away). This source has been proposed to be the heating source of the Orion hot core. Nevertheless, as discussed in $\S 1$, Kaufman et al. (1998) modeled the physical conditions in the Orion hot core, and concluded that an internal energy source is more likely, since the Radio Source "I" is not luminous enough to externally heat the core up to the observed temperatures. Although high resolution molecular line and continuum studies have found evidence of substructure within the core (Migenes et al. 1989; Blake et al. 1996), we apply our spherically symmetric model as a first approximation to this source.

The parameters of our best-fit model are: a mass accretion rate $\dot{M}=1.1 \times 10^{-3} M_{\odot} \mathrm{yr}^{-1}$, a B3 central star, an outer radius of the envelope $R_{\text {core }}=0.025 \mathrm{pc}$, and an opacity index $\beta=1.6$ (see Table 2). The mass accretion rate is determined mainly by the $\mathrm{mm}$ and submm data. The spectral type of the star is constrained by the measured flux density at $30 \mu \mathrm{m}$ (Wynn-Williams et al. 1984), since an spectral type earlier than B3 would have too much flux at this wavelength. Furthermore, the convolved image of the model at $1.3 \mathrm{~mm}$ has a FWHM of $\sim 3^{\prime \prime}$, as observed by Blake et al. (1996; Figure 2, top panel). The total luminosity given by our model is $L_{\text {core }}=2.5 \times 10^{4} L_{\odot}$, being most of this due to the accretion. It is worth noting that this value exceeds the minimum luminosity required by the models of Kaufman et al. (1998) to explain the observed high temperature in the Orion hot core. 


\subsection{IRAS $23385+6053$}

IRAS $23385+6053$ has a quite broad data set available, spanning from centimeter to near infrared wavelengths (see M98). No emission is detected at centimeter wavelengths above 3- $\sigma$ level of $\sim 0.5 \mathrm{mJy}$. At $3.4 \mathrm{~mm}$, M98 detected a compact source with a deconvolved angular size of $4 . " 5 \times 33^{\prime \prime} 6$ (or $0.048 \mathrm{pc}$ for an assumed distance of $4.9 \mathrm{kpc}$ ). They estimated a bolometric luminosity for this source $L \sim 1.6 \times 10^{4} L_{\odot}$. They also detected a compact outflow in $\mathrm{SiO}$ and $\mathrm{HCO}^{+}$lines that is centered on the millimeter source.

In order to explain the lack of a detectable HII region associated with this source, M98 consider two possibilities: a heavily obscured B0 star with residual accretion, or a proto-star undergoing very intense accretion with $\dot{M}=10^{-3} M_{\odot} \mathrm{yr}^{-1}$. For the latter case, using the relation found by Stahler et al. (1986), discussed in $\S 2.5$, they proposed a central proto-star with $M_{*}=39 M_{\odot}$ and $R_{*}=70 R_{\odot}$, and assumed that the observed luminosity is dominated by the accretion luminosity.

Our best-fit (model I) to the observed spectrum is shown in the lower right panel of Figure 3 and upper left panel of Figure 4. This model I (whose parameters are given in Table 2) has a central B2 star, an accretion rate of $10^{-3} M_{\odot} \mathrm{yr}^{-1}$, an external radius $R_{\text {core }}=0.1 \mathrm{pc}$, and an opacity index $\beta=1.6$. The physical properties of this model are given in Table 3 . The lower left panel of Figure 4 shows the intensity profile at $3.4 \mathrm{~mm}$ predicted by our model and the observed intensity profiles along the major and minor axes of the $3.4 \mathrm{~mm}$ map given in Figure 1 of M98 (dot-dashed line). As can be seen in the figure, the agreement is very good. The intensity profile of the model was obtained by convolving (in two dimensions) the intensity distributions at 3.4 $\mathrm{mm}$ given by the model with a Gaussian beam of FWHM $=33^{\prime \prime} 8$ (corresponding to the size of the beam in the observations of M98). Note that no fit was done to the observed intensity profile at $3.4 \mathrm{~mm}$; only the observed flux densities (that are integrated values, with no information on the spatial distribution) were fitted with a model spectrum (upper panel of Fig. 4). It is remarkable that the predicted $3.4 \mathrm{~mm}$ model intensity profile, obtained from the parameter set that provide the best-fit to the spectrum, is in such a good agreement with the observed intensity profile (lower panel of Fig. 4).

The right panels of Figure 4 shows the predictions for an alternative HMC model, similar to the one proposed by M98 (model II), namely a core with a central proto-star with $M_{*}=39 M_{\odot}$, $R_{*}=70 R_{\odot}$, and $L_{\text {core }}=L_{\text {acc }}$. This model has $\dot{M}=1.6 \times 10^{-3} M_{\odot} \quad \mathrm{yr}^{-1}, \mathrm{R}_{\text {core }}=0.19 \mathrm{pc}$ and $\beta=2$. Even though the observed spectrum can be fitted by this model, the predicted intensity profile at $3.4 \mathrm{~mm}$ is too broad and its peak intensity is too low to be detectable above the rms noise of the map (see lower right panel in Fig. 4). This difference is due the large value of $R_{\text {core, with }}$ respect to that of Model I. Therefore, this model appears to be in conflict with the observations. We conclude that a B2 star is most likely embedded in the IRAS $23385+6053$ core, as indicated by model I (Table 2 and 3 and left panel of Fig 4 ). 


\subsection{Temperature Profiles of HMC models}

Figure 5 shows the temperature profiles for the models presented in Figure 3 (from $R_{\mathrm{d}}$ to $\left.R_{\text {core }}\right)$. The different sources are labeled in each panel. These temperature profiles are discontinuous at the photospheric radius, $R_{\mathrm{ph}}$, because the dilution factor, $W\left(R_{\mathrm{ph}}\right)=1 / 2$, and, in our approximation, eqn. (5) then gives $T<T_{\mathrm{ph}}$ at this radius. Instead, there should be a smooth transition between the optically thin and the optically thick regimes and hence a smooth temperature profile. With our simplified treatment for the temperature profile, the difference between the total luminosity of the spectra of model and the input luminosity, $L_{\text {core }}$, is less than $15-20 \%$. It can be seen that the temperature distributions in the optically thick region have a steeper dependence with radius than in the optically thin region, as discussed by Adams \& Shu (1985) and Kenyon et al. (1993). Finally, for all cases, at the external radius, $R_{\text {core }}$, the temperatures are $T>30 K$.

\section{DISCUSSION}

\subsection{General Discussion}

The model studied here, of an envelope infalling onto a central massive star, reproduces very well the currently available observational data for the sources selected in our sample. We have explored two possible density distributions for the structure of the infalling envelope and concluded that a SLS collapse density distribution provides a better agreement with the available observational data, than a SIS density distribution. In order to fit the observations of these sources, our model requires massive, early B-type central stars $\left(10-16 M_{\odot}\right)$, with high values of the mass accretion rate $\left(\sim 10^{-3} M_{\odot} \mathrm{yr}^{-1}\right)$, and core radii $\lesssim 0.1 \mathrm{pc}$. The values of the mass accretion rate obtained exceed by a large factor the "critical" value required to quench the development of a detectable UCHII region, which for an early B-type central star (the proposed central stars), is $\lesssim 10^{-7} M_{\odot} \mathrm{yr}^{-1}$. Therefore, the model lacks a detectable HII region. Furthermore, very small flux densities from dust thermal emission are predicted at centimeters wavelengths.

The values that we have obtained for the radius of the cores are roughly consistent with those inferred from the observations. However, a detailed comparison is not yet possible, since most of the observations give only upper limits for the source sizes. Only in the case of the $3.4 \mathrm{~mm}$ map of IRAS 23385+605 (M98), was it possible to compare the intensity distribution predicted by the model with that observed, and we found a remarkably good agreement with the observed total flux, source size and peak intensity. Additional maps of good $\mathrm{S} / \mathrm{N}$ and high angular resolution are required for a larger number of sources and wavelengths. In this manner, continuum intensity profiles may be able to discriminate between possible models. Similarly, high angular resolution FIR images are needed. Our model predicts a peak flux density $>10^{3} \mathrm{Jy}$ at $\sim 100 \mu \mathrm{m}$. Thus, infrared fluxes around this wavelength, obtained with higher angular resolution than currently 
available to avoid contamination with nearby objects, would be specially relevant in order to further constrain the parameters of the modeled sources.

The intrinsic infrared colors [100-60], [60-25] and [25-12] predicted by our models (see Table 4) are very different from those of observed UCHII regions, $[100-60]=0.26,[60-25]=0.87$, and $[25-12]=0.91$ (Wood \& Churchwell 1989). In particular, a color criterion [60-25] > 1.3 could be use to find isolated HMCs, when their fluxes are not contaminated by nearby sources.

Furthermore, for all the sources, the accretion luminosity clearly exceed, the stellar luminosity (see Table 3). This result points out the relevance of the energy released in the accretion process at this early evolution stage, a basic ingredient that should not be ignored when modeling this kind of object.

\subsection{Radiation Pressure Onto Dust Grains}

The radiation pressure onto dust grains imposes an upper limit to the amount of mass that can accrete onto a massive star. It is therefore important to assess whether or not this effect can halt the collapse of the envelopes that we consider in our models. Kahn (1974) found that the radiation pressure of the diffuse infrared radiation field inside a dusty envelope around a massive proto-star decelerates the flow allowing a maximum luminosity to mass ratio $L / M_{*}=5436 L_{\odot} / M_{\odot}$, although this number depends on the properties of the dust grains. Wolfire \& Cassinelli (1987; hereafter WC87) revised the calculations of the maximum mass that an accreting proto-star can accumulate taking into account updated properties of the interstellar grains. Jijina \& Adams (1996) reexamined this problem including the effect of angular momentum. In this section, we ensure that our models of hot cores fulfill the general constraint that guarantee that inflow will proceed towards the central proto-star.

At the outer boundary of the inflow, the outward radiative acceleration must be less than the inward gravitational acceleration of the gas. This condition can be written as (WC87, eqn. [10])

$$
\Gamma=\frac{\kappa_{\mathrm{H}}^{\mathrm{pr}} L_{\text {core }}}{4 \pi c G M\left(R_{\text {core }}\right)}<1,
$$

where $\kappa_{\mathrm{H}}^{\mathrm{pr}}$ is the flux mean radiation pressure coefficient, $c$ is the speed of the light, $M\left(R_{\text {core }}\right)$ is the total mass inside $R_{\text {core }}$ (star plus core mass), and $L_{\text {core }}=L_{*}+L_{\text {acc }}$ is the total luminosity available to heat and push the core material. Our models have $L_{\text {core }} \lesssim 6 \times 10^{4} L_{\odot}$, typical core masses $M\left(R_{\text {core }}\right) \sim 130 M_{\odot}$, and we approximate the flux mean opacity with the dust Planck mean opacity evaluated at the photospheric temperature. The photospheric temperatures we find are $T_{\mathrm{ph}} \sim 100 \mathrm{~K}$, therefore, $\kappa_{P} \sim 2 \mathrm{~cm}^{2} \mathrm{~g}^{-1}$. For this range of values

$$
\Gamma=0.07\left(\frac{\kappa_{P}}{2 \mathrm{~cm}^{2} \mathrm{~g}^{-1}}\right)\left(\frac{L_{\text {core }}}{6 \times 10^{4} L_{\odot}}\right)\left(\frac{M\left(R_{\text {core }}\right)}{130 M_{\odot}}\right)^{-1}<1,
$$


and the fluid at the outer boundary can flow inward.

On the other side, at the dust destruction front, $R_{\mathrm{d}}$, where the direct stellar radiation is absorbed, the momentum rate of the flow must be greater or equal to the pressure of the stellar radiation field

$$
\dot{M} \geq \frac{L}{u_{\mathrm{d}} c},
$$

where $u_{\mathrm{d}}$ is the velocity of the flow at the dust destruction front, $R_{\mathrm{d}}$. This condition is satisfied for our model $\left(M_{*} \sim 10-16 M_{\odot}, \dot{M} \sim 6 \times 10^{-4}-10^{-3} M_{\odot} \mathrm{yr}^{-1}\right)$ as can be seen in Fig. 5 of WC87. Their figure also shows that our cores have less than the Eddington luminosity, $L_{\text {Edd }}$, where the accretion is halted by radiation pressure on free electrons with an opacity given by Thompson scattering.

Considering an optically thick dusty cocoon plus an optically thin envelope, and assuming an analytic form for the Rosseland mean opacity $\chi_{\mathrm{R}}=\chi_{\mathrm{R} 0}\left(T / T_{s}\right)^{2}$, Kahn (1974) obtained the maximum luminosity to mass ratio $L / M_{*}=5436 L_{\odot} / M_{\odot}$, mentioned above. His eqn. [65] implies that this ratio is inversely proportional both to the cube of the dust sublimation temperature, $T_{\mathrm{sub}}^{3}$, and to $\chi_{R 0}$, the Rosseland mean opacity for radiation at a color temperature $T_{\mathrm{s}}=22,000 \mathrm{~K}$. Kahn used $T_{\text {sub }}=3675 \mathrm{~K}$ and $\chi_{R 0}=600 \mathrm{~cm}^{2} \mathrm{~g}^{-1}$. We find that the sublimation temperature for the grain mixture in our models (see $\S 2.4$ ) is $T_{\text {sub }} \sim 1200 \mathrm{~K}$. Also, the value of $\chi_{R 0}$ must be increased by a factor of $\sim 8$ to agree with our value for the Rosseland mean opacity at $T \sim 1000$ K. These modifications, due to the currently accepted properties of the standard grain mixture used in this work, imply that the maximum luminosity to mass ratio derived by Kahn would currently be $L / M_{*} \sim 20,000 L_{\odot} / M_{\odot}$. This result contrasts with the detailed modeling of the dust destruction process carried out by WC87. They found that very massive stars can only be formed with modifications of the standard MRN grain mixture for accretion onto massive stars with $M_{*}=$ 60, 100 and $200 M_{\odot}$, in which the luminosity to mass ratios are larger than $L / M_{*}=8974 L_{\odot} / M_{\odot}$. On the other hand, Jijina \& Adams (1996) found that the maximum luminosity to mass ratio is less restrictive for a rotating collapse than for the spherical infall. In any case, all our models have luminosity to mass ratios $L_{\text {core }} / M_{*}<4300 L_{\odot} / M_{\odot}$, which implies that accretion can continue onto the central star.

We therefore consider that radiation pressure onto dust grains will not stop the accretion flow onto the central proto-star for the modeled HMCs discussed in this work. For simplicity, we have not considered any deviations of the flow from the dynamics of a logatropic collapsing core.

\subsection{End of the Accretion Phase}

The luminosity to mass ratios of the modeled HMCs discussed in this work are high but still remain below the critical ratios for radiation pressure onto dust grains needed to halt the accretion 
(see $§ 5.2$ ). Nevertheless, this ratio, given by

$$
\frac{L_{\mathrm{core}}}{M_{*}}=\frac{L_{*}}{M_{*}}+\frac{G \dot{M}_{*}}{R_{*}},
$$

will increase with time both because the luminosity of the central star increases with time, and because the mass accretion rate increases as $\dot{M} \propto t^{3}$ (eqn. [1]) in the logatropic model. Therefore, radiation pressure could eventually halt the collapse of matter onto the central star. This is only a transient process since, as the accretion is shut off, so is the accretion luminosity, an important contributor to the total luminosity. Thus, one is tempted to speculate that, if a stellar wind can turn on after the flow is impulsively reversed, the wind could help clear out the accreting flow. An HII region can then be produced, although in this scenario its evolution would be governed by the reversal of the accretion flow, a complex problem worth studying in detail.

If this process occurs on a short time scale compared to the accretion time scale, one can make a simple estimate of the final masses of the central stars inside the HMCs when the accretion flow is halted by radiation pressure. We assume a main sequence mass-luminosity relation, $L_{*} \propto M_{*}^{3}$, and mass-radius relation, $R_{*} \propto M_{*}^{0.73}$ (e.g. Bowers \& Deeming 1984), even though, as discussed in $\S 2.5$, it is not clear if these relations hold for massive stars formed under intense accretion rates which increase steeply with time, considered in this work. Figure 6 shows the luminosity to mass ratio as function of the dimensionless time $t / t_{\text {age }}$, where $t_{\text {age }}$ is the present age of the HMC (solid line). We have assumed a $12.6 M_{\odot}$ central star and a mass accretion rate $\dot{M}=1 \times 10^{-3} M_{\odot} \mathrm{yr}^{-1}$, at the dimensionless time $t / t_{\text {age }}=1$. These values correspond to the model for IRAS $23385+6053$ which has $t_{\text {age }}=5 \times 10^{4}$ yr (see Tables 2 and 3 ). The dot-dashed line is the stellar contribution to the luminosity to mass ratio and the dashed line is the accretion contribution to this ratio. The triangles show the mass of the central star $\left(M_{*} \propto t^{4}\right.$, see eqn. [1]) labeled on the right hand vertical axis. Independently of the exact critical value for the luminosity to mass ratio for radiation pressure to halt the accretion flow (see discussion in $§ 5.2$ ), this figure shows that the evolution of this ratio is very fast so one would expect that in less than $10^{5} \mathrm{yr}$ the accretion of mass onto the central star of the HMC should stop and an ultracompact HII region should develop inside these cores.

Finally, if all OB stars are formed within HMCs under high accretion rates, one can estimate the expected number of HMCs in this phase. Taking the rate of formation of $\mathrm{O}$ stars in the Galaxy $\dot{N} \sim 1 / 300 \mathrm{yr}^{-1}$ (Wood \& Churchwell 1989), and the time in the HMC phase when the central star is a late B star, $\Delta t \sim 10^{5} \mathrm{yr}$, one should expect to find $\sim 300$ HMCs at the present time. On the other hand, the period of time that a core contains an early $\mathrm{O}$ star is very small, $\Delta t \lesssim 2 \times 10^{4} \mathrm{yr}$, so less than 60 HMCs with very luminous massive stars inside should be expected. The number of expected HMCs can increase by a factor of 10 if a higher star formation rate of $\mathrm{O}$ stars, $\sim 4 \times 10^{-2} \mathrm{yr}^{-1}$ (Güsten \& Mezger 1983), is used. 


\section{CONCLUSIONS}

Our main results can be summarized as follows:

1. The observed spectra of several HMCs (G34.24 + 0.13MM, W3 $\left(\mathrm{H}_{2} 0\right)$, the Orion hot core and IRAS $23385+6053)$ can be reproduced by massive envelopes accreting onto young massive central stars. In order to fit the available data, we require early B-type stars with high mass accretion rates, $\dot{M} \gtrsim 6 \times 10^{-4} M_{\odot} \mathrm{yr}^{-1}$, and time-scales $t_{\text {age }} \lesssim 10^{5} \mathrm{yr}$. The main heating agent is the accretion luminosity $\left(\mathrm{L}_{\mathrm{acc}}>L_{*}\right.$; see Table 3$)$.

2. The spectra are strongly dependent on the assumed envelope density profile. Isothermal collapsing envelopes have less mass in the envelope for a given mass accretion rate, with respect to the logatropic collapsing envelopes. Therefore, they require very high accretion rates $\left(>10^{-3} M_{\odot} \mathrm{yr}^{-1}\right)$ in order to reproduce the observed millimeter emission. Nevertheless, high accretion rate SIS models predict strong mid-infrared emission which is, in general, not observed.

3. Models of the structure of massive stars formed under intense mass accretion rates that increase with time, discussed in this work, are necessary to determine the characteristics (e.g. radius, intrinsic luminosity) of this type of object. In particular, we have used central stars with radii larger than the ZAMS radii in order to avoid excess fluxes at mid infrared wavelengths with respect to those observed.

4. High angular resolution mid and far IR observations (which avoid contamination by nearby massive stars) are crucial to constrain the total luminosity of the HMCs.

5. The critical mass accretion rate required to quench the development of an UCHII region is very small compared to the accretion rates required by all the HMC models studied here. Therefore, at this stage the HMCs have no detectable HII region.

6. We speculate that when a critical luminosity to mass ratio is achieved, the infall will be halted by a powerful stellar wind and a detectable UCHII region can appear.

We are greatly indebted with Javier Ballesteros, María Eugenia Contreras, and Rosa Izela Díaz-Miller with whom we originally discussed the work presented here. We also thank Guillem Anglada, Riccardo Cesaroni, Guido Garay, William Henney, Stan Kurtz, Sergio Molinari, Luis F. Rodríguez, Leonardo Testi, Malcom Walmsley and Alan Watson for useful discussions and helpful suggestions. We thank Leonardo Testi for providing us the original data for IRAS 23385+6053. Thanks too to Robert Estalella for providing a program to convolve our results with the observing beam. M. O., S. L. and P. D. acknowledge support from DGAPA/UNAM and CONACYT. S. L. also acknowledges support from the Simon Guggenheim Memorial Foundation. M. O. also 
acknowledges partial support from the Programa de Cooperación Científica con Iberoamérica (Spain). 
Table 1a. Physical Properties of a Grid of SLS Hot Core Models

\begin{tabular}{|c|c|c|c|c|c|c|c|}
\hline Model & $\begin{array}{c}\mathrm{L}_{\text {core }} \\
\left(10^{4} \mathrm{~L}_{\odot}\right)\end{array}$ & $\begin{array}{l}\mathrm{R}_{\mathrm{d}} \\
(\mathrm{AU})\end{array}$ & $\begin{array}{c}\mathrm{R}_{\mathrm{ph}} \\
\left(10^{3} \mathrm{AU}\right)\end{array}$ & $\begin{array}{c}\mathrm{n}\left(R_{\text {core }}\right) \\
\left(10^{5} \mathrm{~cm}^{-3}\right) \\
\end{array}$ & $\begin{array}{l}\mathrm{T}_{\mathrm{ph}} \\
(\mathrm{K})\end{array}$ & $\begin{array}{c}\mathrm{r}_{\mathrm{ew}} \\
\left(10^{3} \mathrm{AU}\right) \\
\end{array}$ & $\begin{array}{l}\mathrm{M}_{\text {core }} \\
\left(\mathrm{M}_{\odot}\right)\end{array}$ \\
\hline \multicolumn{8}{|c|}{$\begin{array}{c}\text { B0 star; } \quad \mathrm{R}_{\text {core }}=0.1 \mathrm{pc} \\
\dot{M}\left(M_{\odot} \mathrm{yr}^{-1}\right)\end{array}$} \\
\hline $1.5 \times 10^{-3}$ & 16 & 118 & 11.0 & 14 & 74 & 21 & 495 \\
\hline $4.4 \times 10^{-4}$ & 6.4 & 47 & 3.2 & 2.6 & 110 & 47 & 86 \\
\hline $8.1 \times 10^{-5}$ & 3.2 & 18 & 0.4 & 0.2 & 260 & 144 & 8 \\
\hline \multicolumn{8}{|c|}{$\begin{array}{c}\mathrm{R}_{\text {core }}=0.1 \mathrm{pc} ; \dot{M}=4.4 \times 10^{-4} M_{\odot} \mathrm{yr}^{-1} \\
\text { Spectral type }\end{array}$} \\
\hline O7 & 15.2 & 57 & 2.3 & 1.1 & 162 & 95 & 36 \\
\hline B0 & 6.4 & 47 & 3.2 & 2.6 & 110 & 47 & 86 \\
\hline B3 & 4.3 & 45 & 4.2 & 4.3 & 87 & 30 & 146 \\
\hline \multicolumn{8}{|c|}{$\begin{array}{c}\text { B0 star; } \dot{M}=4.4 \times 10^{-4} M_{\odot} \mathrm{yr}^{-1} \\
R_{\text {core }}(\mathrm{pc})\end{array}$} \\
\hline 0.15 & 6.4 & $\overline{47}$ & 3.9 & 1.8 & 100 & 47 & 205 \\
\hline 0.10 & 6.4 & 47 & 3.2 & 2.6 & 110 & 47 & 86 \\
\hline 0.05 & 6.4 & 47 & 2.3 & 4.3 & 130 & 47 & 19 \\
\hline
\end{tabular}

Table 1b. Physical Properties of a Grid of SiS Hot Core Models

\begin{tabular}{|c|c|c|c|c|c|c|c|}
\hline Model & $\begin{array}{c}\mathrm{L}_{\text {core }} \\
\left(10^{4} \mathrm{~L}_{\odot}\right)\end{array}$ & $\begin{array}{l}\mathrm{R}_{\mathrm{d}} \\
(\mathrm{AU})\end{array}$ & $\begin{array}{c}\mathrm{R}_{\mathrm{ph}} \\
\left(10^{3} \mathrm{AU}\right)\end{array}$ & $\begin{array}{c}\mathrm{n}\left(R_{\text {core }}\right) \\
\left(10^{5} \mathrm{~cm}^{-3}\right)\end{array}$ & $\begin{array}{l}\mathrm{T}_{\mathrm{ph}} \\
(\mathrm{K})\end{array}$ & $\begin{array}{c}\mathrm{r}_{\mathrm{ew}} \\
\left(10^{3} \mathrm{AU}\right)\end{array}$ & $\begin{array}{l}\mathrm{M}_{\text {core }} \\
\left(\mathrm{M}_{\odot}\right)\end{array}$ \\
\hline \multicolumn{8}{|c|}{$\begin{array}{c}\text { B0 star; } \quad \mathrm{R}_{\text {core }}=0.1 \mathrm{pc} \\
\dot{M}\left(M_{\odot} y r^{-1}\right)\end{array}$} \\
\hline $1.5 \times 10^{-3}$ & 16 & 115 & 6.5 & 2.3 & 98 & 4.1 & 141 \\
\hline $4.4 \times 10^{-4}$ & 6.4 & 47 & 2.1 & 1.0 & 136 & 9.4 & 47 \\
\hline $8.1 \times 10^{-5}$ & 3.2 & 18 & 0.3 & 0.1 & 284 & 29 & 4 \\
\hline \multicolumn{8}{|c|}{$\begin{array}{c}\mathrm{R}_{\text {core }}=0.1 \mathrm{pc} ; \dot{M}=4.4 \times 10^{-4} M_{\odot} \mathrm{yr}^{-1} \\
\text { Spectral type }\end{array}$} \\
\hline O7 & 15.2 & 56 & 1.6 & 0.9 & 192 & 19.0 & 20 \\
\hline B0 & 6.4 & 47 & 2.1 & 1.0 & 136 & 9.4 & 47 \\
\hline B3 & 4.3 & 44 & 2.5 & 1.0 & 114 & 6.0 & 57 \\
\hline \multicolumn{8}{|c|}{$\begin{array}{c}\text { B0 star; } \dot{M}=4.4 \times 10^{-4} M_{\odot} \mathrm{yr}^{-1} \\
R_{\text {core }}(\mathrm{pc})\end{array}$} \\
\hline 0.15 & 6.4 & 47 & 2.3 & 0.4 & 130 & 9.3 & 84 \\
\hline 0.10 & 6.4 & 47 & 2.1 & 1.0 & 136 & 9.4 & 47 \\
\hline 0.05 & 6.4 & 47 & 1.6 & 4.0 & 157 & 9.4 & 11 \\
\hline
\end{tabular}


Table 2. Model Parameters Determined from Spectral Fits to Individual Sources

\begin{tabular}{ccccccc}
\hline \hline Source & $\begin{array}{c}M_{*}^{a} \\
\left(\mathrm{M}_{\odot}\right)\end{array}$ & $\begin{array}{c}L_{*}^{b} \\
\left(\mathrm{~L}_{\odot}\right)\end{array}$ & $\begin{array}{c}\dot{M} \\
\left(\mathrm{M}_{\odot} \mathrm{yr}^{-1}\right)\end{array}$ & $\begin{array}{c}R_{\mathrm{core}} \\
(\mathrm{pc})\end{array}$ & $\begin{array}{c}D^{c} \\
(\mathrm{kpc})\end{array}$ \\
\hline G34.24+0.13MM & 10.0 & $1.0 \times 10^{3}$ & $6.5 \times 10^{-4}$ & 0.07 & 2.0 & 3.7 \\
W3 $\left(\mathrm{H}_{2} \mathrm{O}\right)$ & 15.8 & $2.5 \times 10^{4}$ & $1.2 \times 10^{-3}$ & 0.05 & 1.6 & 2.2 \\
Orion Hot Core & 10.0 & $1.0 \times 10^{3}$ & $1.1 \times 10^{-3}$ & 0.02 & 1.6 & 0.5 \\
IRAS 23385+6053 & 12.6 & $2.8 \times 10^{3}$ & $1.0 \times 10^{-3}$ & 0.10 & 1.6 & 4.9 \\
\hline
\end{tabular}

${ }^{\mathrm{a}} R_{*}=10^{12} \mathrm{~cm}$ is assumed.

b Taken from Thompson (1984), for a given $M_{*}$.

c The adopted distances are taken from Kuchar \& Bania 1994, Humphreys 1978, Genzel et al. 1981, and Molinari et al. 1998.

Table 3. Physical Properties of Best-Fit Models to Individual HMCs

\begin{tabular}{|c|c|c|c|c|c|c|c|c|c|c|}
\hline Source & $\begin{array}{c}R_{\mathrm{d}} \\
(\mathrm{AU})\end{array}$ & $\begin{array}{c}R_{\mathrm{ph}} \\
\left(10^{3} \mathrm{AU}\right)\end{array}$ & $\begin{array}{l}T_{\mathrm{ph}} \\
(\mathrm{K})\end{array}$ & $\begin{array}{c}n\left(R_{\text {core }}\right) \\
\left(10^{6} \mathrm{~cm}^{-3}\right)\end{array}$ & $\begin{array}{c}L_{\text {core }} \\
\left(10^{4} \mathrm{~L}_{\odot}\right)\end{array}$ & $\begin{array}{c}L_{\mathrm{acc}} / L_{\text {core }} \\
(\%)\end{array}$ & $\begin{array}{l}M_{\text {core }} \\
\left(\mathrm{M}_{\odot}\right)\end{array}$ & $\begin{array}{c}N\left(H_{2}\right) \\
\left(10^{24} \mathrm{~cm}^{-2}\right)\end{array}$ & $\begin{array}{c}t_{\text {age }} \\
\left(10^{4} \mathrm{yr}\right)\end{array}$ & $\begin{array}{c}r_{\text {ew }} \\
\left(10^{3} \mathrm{AU}\right)\end{array}$ \\
\hline G34.24+0.13MM & 35 & 3.8 & 72 & 1.0 & 1.6 & 94 & 131 & 34 & 6.1 & 20 \\
\hline $\mathrm{W} 3\left(\mathrm{H}_{2} \mathrm{O}\right)$ & 74 & 5.3 & 87 & 2.0 & 6.7 & 62 & 83 & 48 & 5.3 & 24 \\
\hline Orion hot core & 52 & 3.2 & 88 & 5.0 & 2.5 & 96 & 27 & 55 & 3.7 & 16 \\
\hline IRAS $23385+6053$ & 54 & 7.0 & 62 & 1.1 & 3.1 & 91 & 382 & 47 & 5.0 & 21 \\
\hline
\end{tabular}


Table 4. Infrared Colors of the Best-Fit Models

\begin{tabular}{lccc}
\hline \hline \multicolumn{1}{c}{ Source } & {$[100-60]$} & {$[60-25]$} & {$[25-12]$} \\
\hline G34.24+0.13MM & 0.04 & 2.32 & 4.60 \\
W3 $\left(\mathrm{H}_{2} \mathrm{O}\right)$ & -0.18 & 1.36 & 3.58 \\
Orion hot core & -0.16 & 1.27 & 3.43 \\
IRAS 23385+6053 & 0.20 & 2.86 & 5.84 \\
& & & \\
\hline
\end{tabular}




\section{REFERENCES}

Alcolea, J., Menten, K. M., Moran, J. M. \& Reid, M. J. 1992, in Astrophysical Masers, ed. A. W. Clegg \& G. E. Nedoluha (Berlin: Springer-Verlag), 225

Adams, F. C., Lada, C. J. \& Shu, F. H. 1987, ApJ, 312, 788

Adams, F. C., \& Shu, F. H. 1985, ApJ, 296, 655

Adams, F. C., \& Shu, F. H. 1986, ApJ, 308, 836

Beech, M., \& Mitalas, R. 1994, ApJS, 95, 517

Bernasconi, P. A. \& Maeder, A. 1996, A\&A, 307, 829

Blake, G. A., Mundy, L. G., Carlstrom, J. E., Padin, S., Scott, S. L., Scoville, N. Z., \& Woody, D. P. 1996, ApJ, 472, L49

Bowers, R., \& Deeming, T. 1984, Astrophysics I, Stars (Boston: Jones and Bartlett Publishers, Inc.)

Cesaroni, R., Churchwell, E., Hofner, P., Walmsley, C. M.\& Kurtz, S. 1994a, A\&A, 288, 903

Cesaroni, R., Olmi, L., Walmsley, C. M., Churchwell, E.\& Hofner, P. 1994b, ApJ, 435, L137

Chandler, C. J.\& Wood, D. O. S. 1997, MNRAS, 287, 445

D’Alessio, P. 1996, Ph.D. Thesis, Universidad Nacional Autónoma de México

Genzel, R., \& Stutzki, J. 1989, ARA\&A, 27, 41

Genzel, R., Reid, M.J., Moran, J.M., \& Downes, D. 1981, ApJ, 244, 884

Gómez, Y., Garay, G., \& Lizano, S. 1995, ApJ453, 727

Güsten, R., \& Mezger, P. G. 1983 Vistas in Astronomy, 26, 159

Hofner, P., Kurtz, S., Churchwell, E., Walmsley, C. M., \& Cesaroni, R. 1996, ApJ, 460, 359

Hunter, T. R., Neugebauer, G., Benford, D. J., Matthews, K., Lis, D. C., Serabyn, E., \& Phillips, T. G. 1998, ApJ, 493, L97 (H98)

Humphreys, R. M. 1978, ApJS, 38, 309

Jijina, J., \& Adams, F. C. 1996, ApJ, 462, 874

Kahn, F. D. 1974, A\&A, 37, 149

Kaufman, M. J., Hollenbach, D. J. \& Tielens, A.G.G.M. 1998, ApJ, 497, 276

Kenyon, S., Calvet, N., \& Hartmann, L. 1993, ApJ, 414, 676

Kuchar, T. A., \& Bania, T. M. 1994, ApJ, 436, 117

Kurtz, S., Cesaroni, R., Churchwell, E., Hofner, P., \& Walmsley, C. M. 1999, in Protostars \& Planets IV, ed. V. Mannings, A. P. Boss, \& S. S. Russell (Tucson: Univ. of Arizona Press), in press.

Lamy, Ph. L. 1974, A\&A, 35, 197 
Lizano, S., \& Shu, F. H. 1989, ApJ, 342, 834

Mathis, J. S., Rumpl, W., \& Nordsieck, K. H. 1977, ApJ, 217, 425

Masson, C. R., \& Mundy, L. G. 1988, ApJ, 324, 538

McLaughlin, D. E.,\& Pudritz, R. E. 1996, ApJ, 469, 194

McLaughlin, D. E.,\& Pudritz, R. E. 1997, ApJ, 476, 750

Mezger, P. G., Wink, J. E., \& Zylka, R. 1990, A\&A, 228, 95

Migenes, V., Johnston, K. J., Pauls, T. A., \& Wilson, T. L. 1989, ApJ, 347, 294

Mihalas, D. 1978, Stellar Atmospheres (New York: Freeman, 2nd edition)

Molinari, S., Testi, L., Brand, J., Cesaroni, R., \& Palla, F. 1998, ApJ, 505, L39 (M98)

Murata, Y., Kawabe, R., Ishiguro, M., Hasegawa, T., \& Hayashi, M. 1991, in IAU Symp. 147, Fragmentation of Molecular Clouds and Star Formation, ed. E. Falgarone, F. Boulanger, \& G. Duvert (Dordrecht: Kluwer), 357

Myers, P. C.,\& Fuller, G. A. 1992, ApJ, 396, 631

Reid, M. J., Argon, A. L., Masson, C. R., Menten, K. M. \& Moran, J. M. 1995, ApJ, 443, 238

Sandford, S. A. \& Allamandolla, L. J. 1993, ApJ, 417, 815

Shu, F. H. 1977, ApJ, 214, 488

Stahler, S. W., Shu, F. H., \& Taam, R. E. 1980, ApJ, 241, 637

Stahler, S. W., Palla, F., \& Salpeter, E. E. 1986, ApJ, 302, 590

Testi, L., Felli, M., Persi, P., \& Roth, M. 1998, A\&A, 329, 233 (T98)

Thompson, R. I. 1984, ApJ, 283, 165

Turner, J. L. \& Welch, W. J. 1984, ApJ, 287, L81

Vacca, W. D., Garmany, C. D. \& Shull, J. M. 1996, ApJ, 460, 914

Walmsley, C. M. 1995, RevMexAA Serie de Conf., 1, 137

Wilner, D. J., Welch, W. J. \& Forster, J. R. 1995, ApJ, 449, L73

Wilner, D. J., Reid, M. J. \& Menten, K. M. 1999, ApJ, 513, 775

Wilson, T. L., Gaume, R. A. \& Johnston, K. J. 1993 ApJ, 402, 230

Wink, J. E., Duvert, G., Guilloteau, S., Gusten, R., Walmsley, C. M. \& Wilson, T. L. 1994, A\&A, 281,505

Wolfire, M. G., \& Cassinelli, J. P. 1987, ApJ, 319, 850 (WC87)

Wood, D. O. S. \& Churchwell, E. 1989, ApJ, 340, 265

Wright, M. C. H., Sandell, G., Wilner, D. J. \& Plambeck, R. L. 1992, ApJ, 393, 225

Wright, M. C. H., Plambeck R. L. \& Wilner, D. J. 1996, ApJ, 469, 216 
Winn-Williams, C. G., Genzel, R., Becklin, E. E., \& Downes, D. 1984, ApJ, 281172

Wyrowski, F., Hofner, P., Schilke, P., Walmsley, C. M., Wilner, D. J. \& Wink, J. E. 1997, A\&A, 320, L17

Wyrowski, F., Schilke, P., Walmsley, C. M., \& Menten, K. 1999, ApJ, 514, L43

Yorke, H. 1984, Workshop on Star Formation, ed. R. D. Wolstencroft (Edinburgh: Royal Observatory), p. 63 
Fig. 1.- (a) Spectra for different sets of the SLS collapse density distribution HMC models. The top panel shows the trends of the spectra obtained for three different values of the mass accretion rate, for a $\mathrm{B} 0$ central star and an external radius $R_{\text {core }}=0.1 \mathrm{pc}$. The middle panel shows models with different spectral types of the central star, for a given external radius $R_{\text {core }}=0.1 \mathrm{pc}$ and accretion rate of $\dot{M}=4.4 \times 10^{-4} M_{\odot} \mathrm{yr}^{-1}$. The bottom panel shows models with different external radii of the envelope, for a central B0 star and an accretion rate $\dot{M}=4.4 \times 10^{-4} M_{\odot} \mathrm{yr}^{-1}$. (b) Same as 1(a) but for the SIS collapse density distribution HMC models. In all cases, a distance of $4.9 \mathrm{kpc}$ were adopted.

Fig. 2.- Observed flux densities of the source G34.24+0.13MM (Hunter et al. 1998; Goss 1999, personal communication). Three modeled spectra with $R_{\text {core }}=0.07 \mathrm{pc}$ and a central B3 star $\left(10 M_{\odot}\right)$ are also shown. The dotted line corresponds to the collapse density distribution of the SIS, with $\dot{M}=1.0 \times 10^{-3} M_{\odot} \mathrm{yr}^{-1}$, and the dot-dashed line corresponds to the collapse density distribution of the SLS, with $\dot{M}=6.5 \times 10^{-4} M_{\odot} \mathrm{yr}^{-1}$, when ZAMS radii are adopted. The solid line shows the spectrum of the SLS model but with a star that has a large radius, $R_{*}=10^{12} \mathrm{~cm}$.

Fig. 3.- Observed flux densities for the sources G34.24+0.13MM (Hunter et al. 1998; Goss 1999, personal communication), W3 $\left(\mathrm{H}_{2} \mathrm{O}\right)$ (Wyrowski et al. 1997; Wilner et al. 1995), Orion hot core (Wright et al. 1992; Murata et al. 1991; Masson et al. 1985; Mezger et al. 1990; The fluxes and errorbars at $30 \mu \mathrm{m}$ and $1.3 \mathrm{~mm}$ was estimated from the maps of Wynn-Williams et al. 1984 and Blake et al. 1996, respectively), and IRAS 23385+6053 (Molinari et al. 1998; IRAS-PSC2), and model spectra. The parameters of each model are listed in Table 2.

Fig. 4.- Observed flux densities (Molinari et al. 1998; IRAS-PSC2) and two models for the source IRAS $23385+6053$. The upper left panel shows the spectrum for a model I, with B2 central star, $\dot{M}=10^{-3} M_{\odot} \mathrm{yr}^{-1}, R_{\text {core }}=0.1 \mathrm{pc}$, and $\beta=1.6$. The lower left panel shows the intensity profile of the $3.4 \mathrm{~mm}$ emission given by the model (solid line) and the observed intensity profiles along the major and minor axes of the $3.4 \mathrm{~mm}$ map given in Fig. 1 of M98 (dot-dashed lines). The upper right and lower right panels correspond to model II with $\dot{M}=1.6 \times 10^{-3} M_{\odot} \mathrm{yr}^{-1}, R_{\text {core }}=0.19 \mathrm{pc}$, and $\beta=2$, and a central proto-star with $M_{*}=39 M_{\odot}$ and $R_{*}=70 R_{\odot}$ (for this model, $L_{\text {core }}=L_{\text {acc }}$ and $L_{*}$ is neglected)

Fig. 5.- Temperature profiles in the range $R_{\mathrm{d}}<r<R_{\text {core }}$, for the models shown in Figure 3 . The different sources are labeled in each panel. The parameters of each model are given in Table 2. The dust destruction radius, $R_{\mathrm{d}}$, the photospheric radius, $R_{\mathrm{ph}}$, and the photospheric temperature, $T_{\mathrm{ph}}$, for each source are given in Table 3 .

Fig. 6. - Luminosity to mass ratio as function of the dimensionless time $t / t_{\text {age }}$ (solid line). The dotdashed line is the stellar contribution to this ratio and the dashed line is the accretion contribution. The triangles show the mass of the central star, labeled on the right hand vertical axis. 



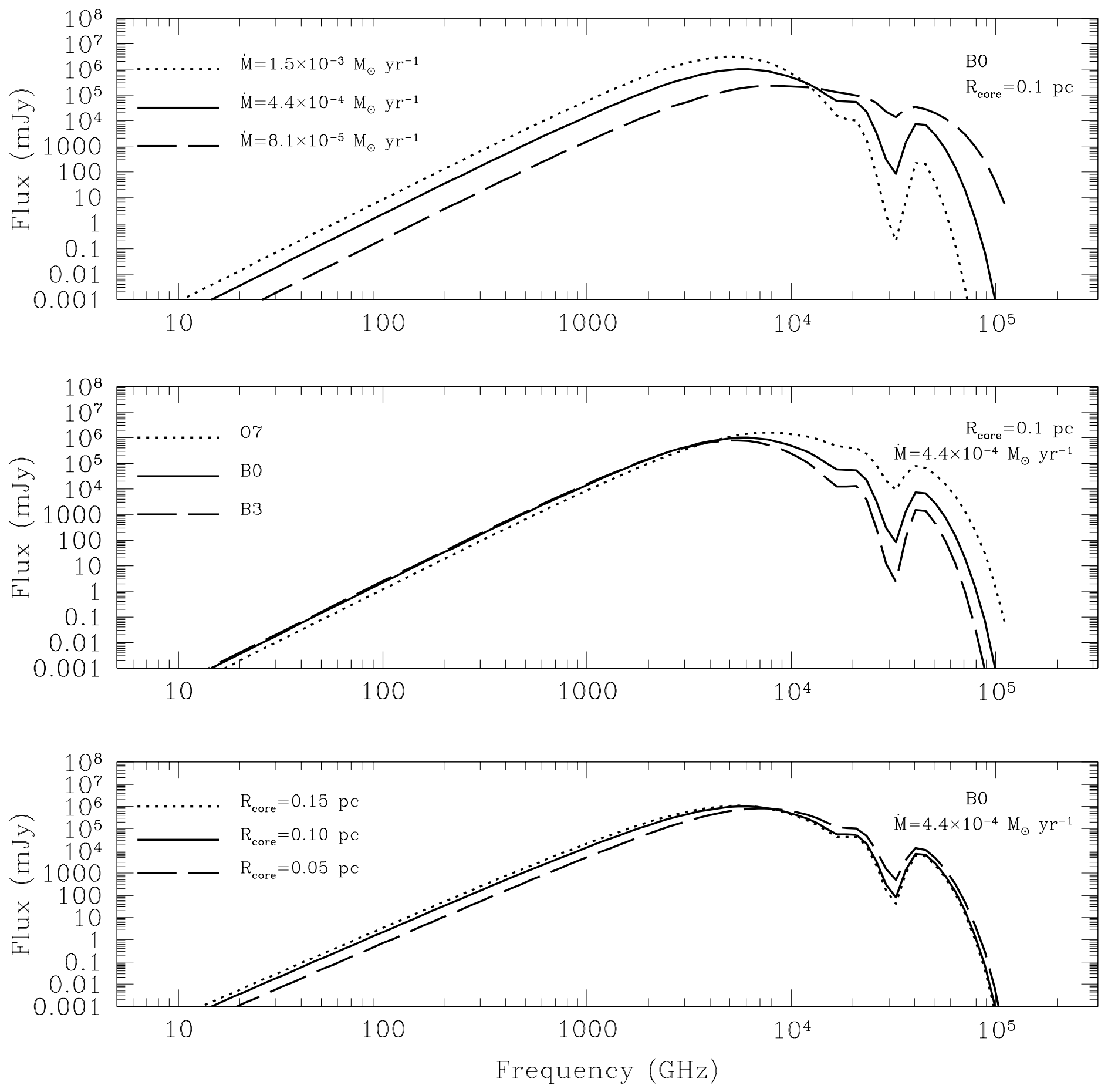


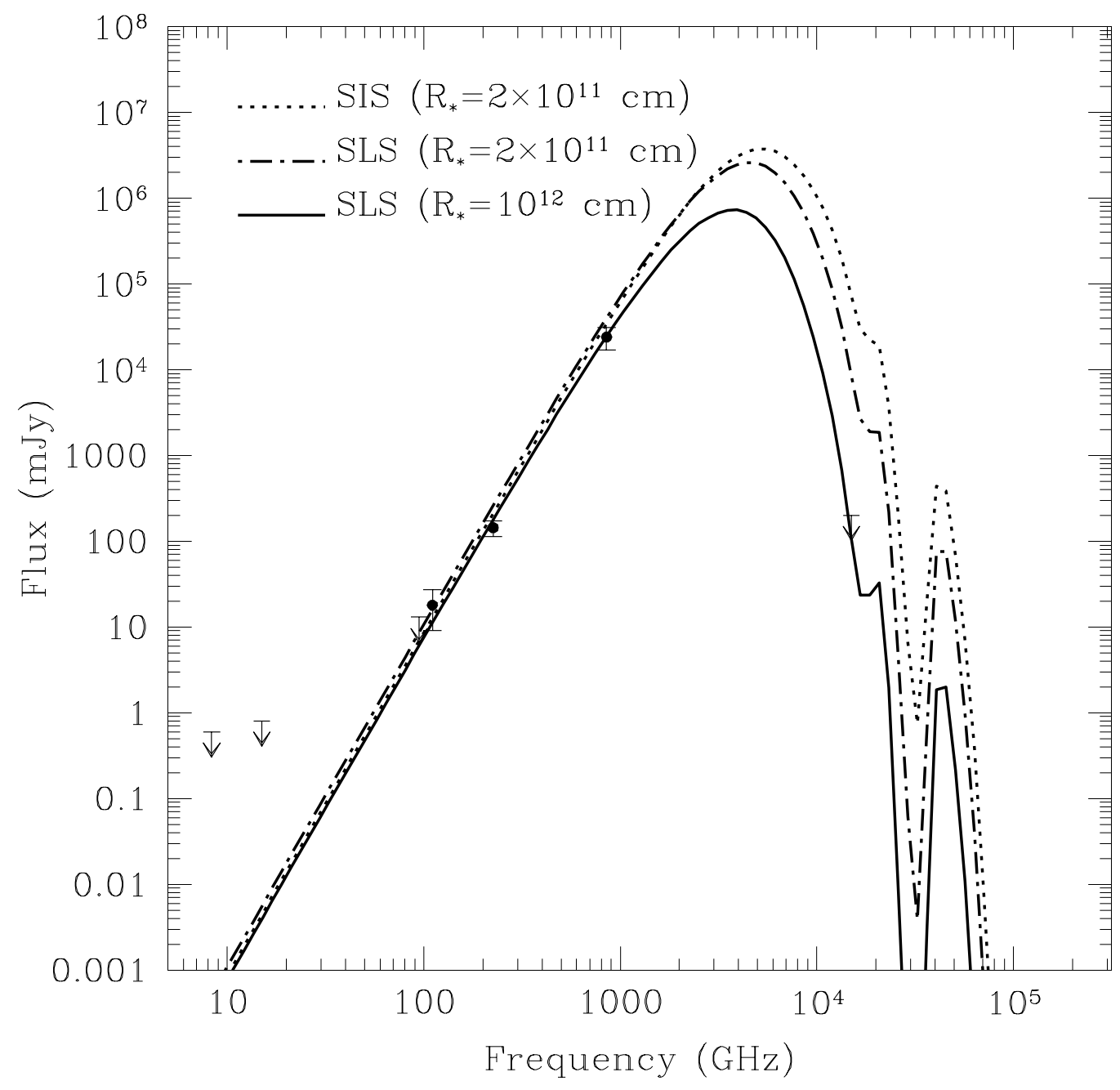



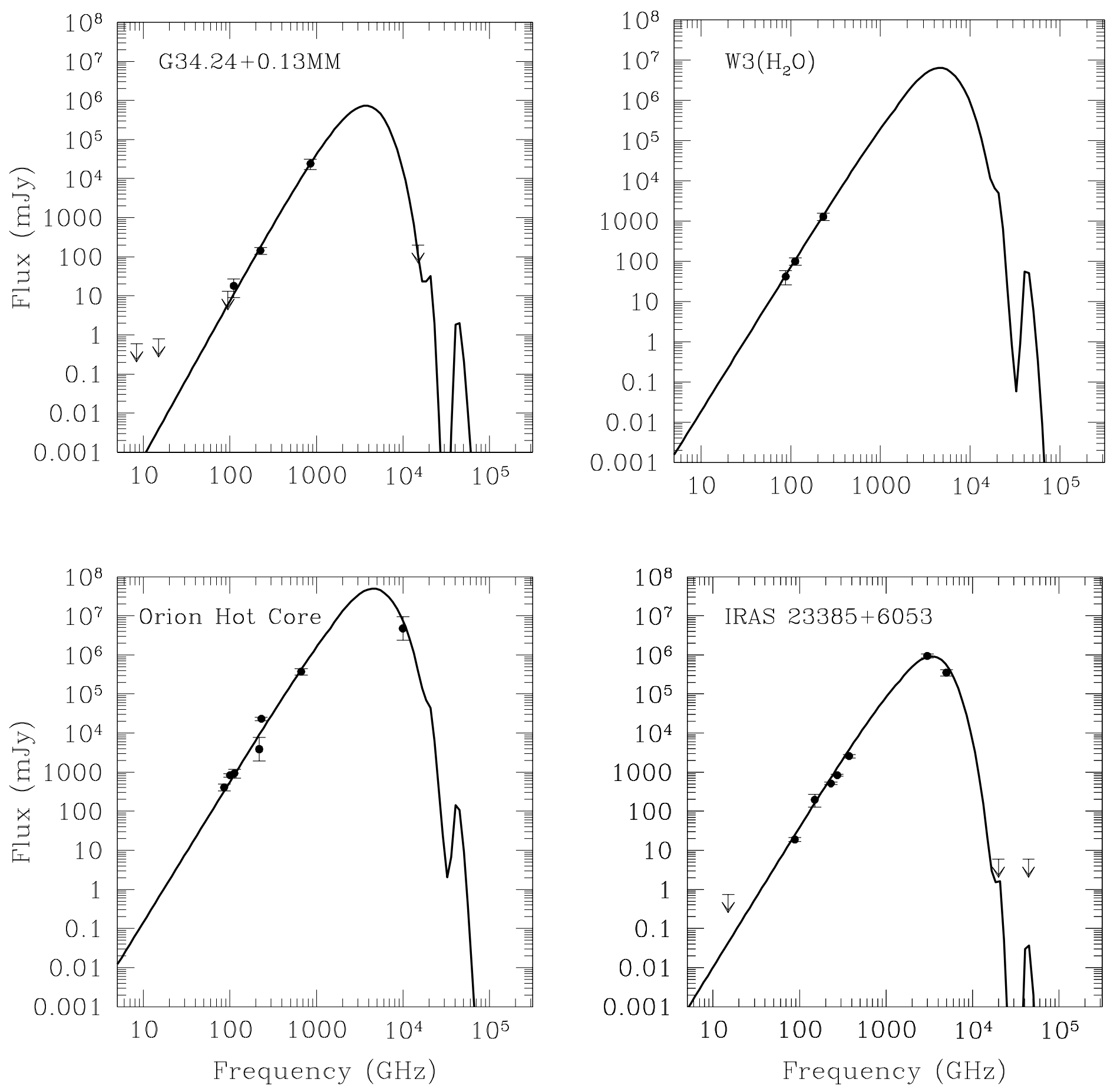

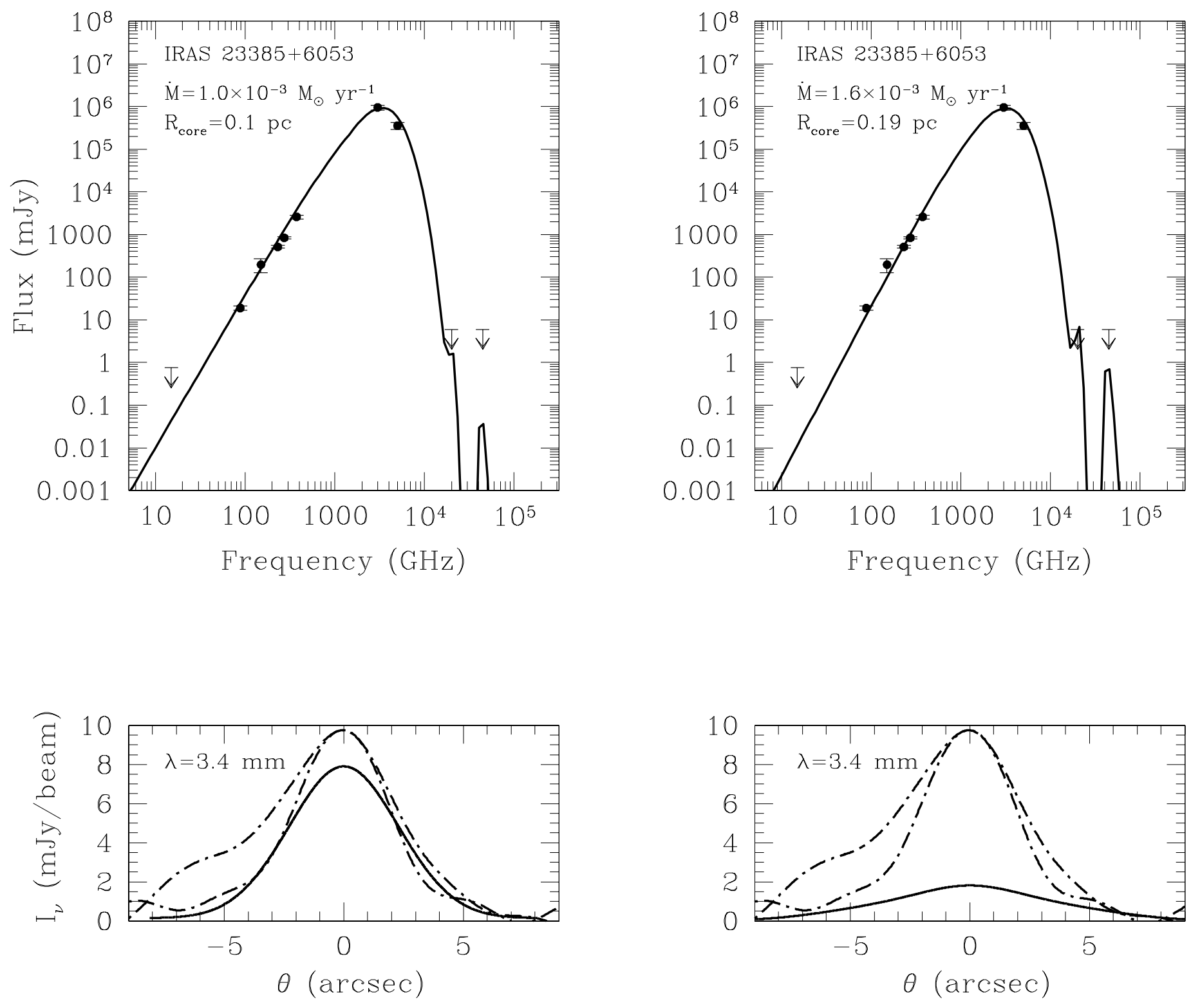

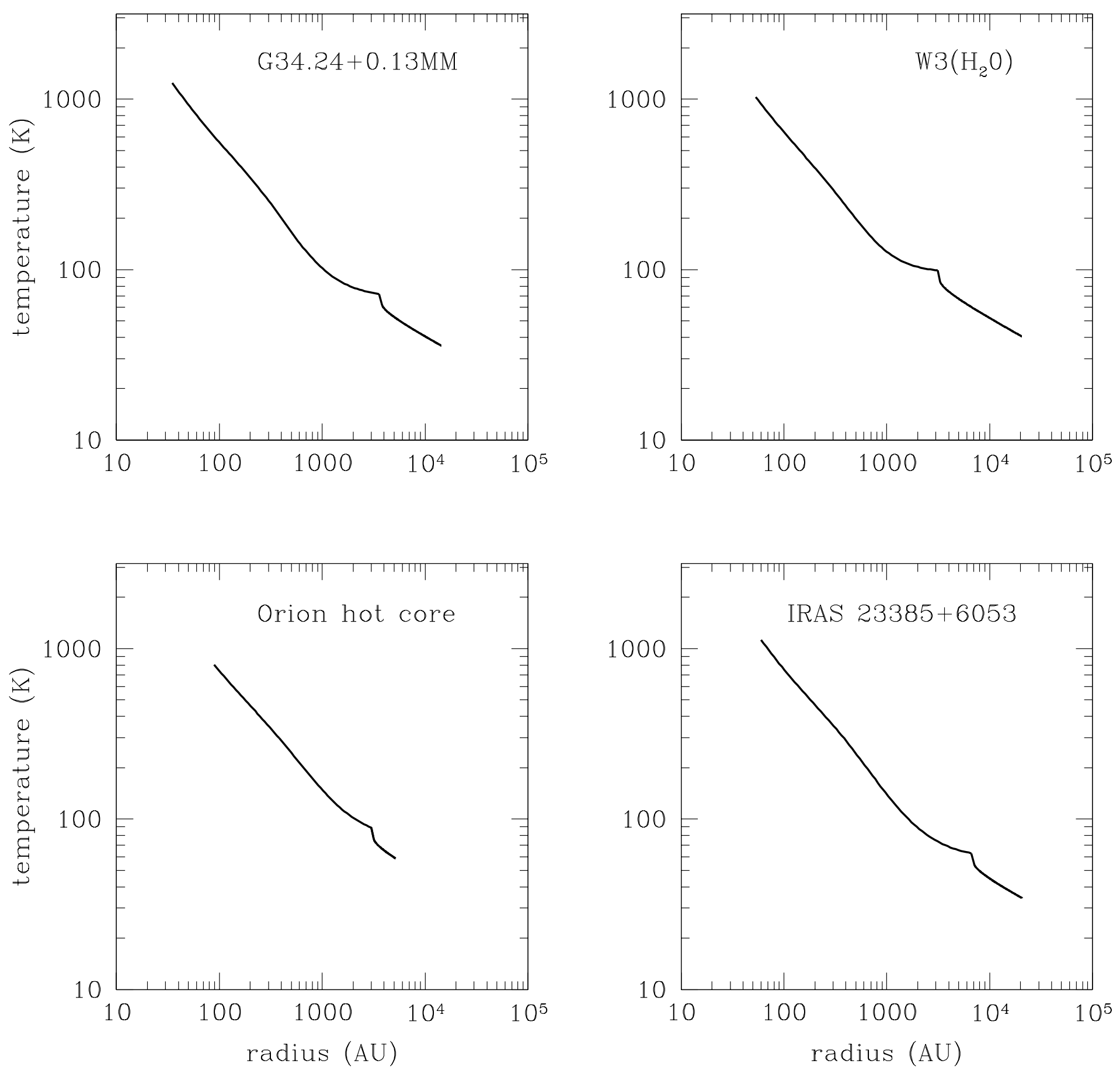


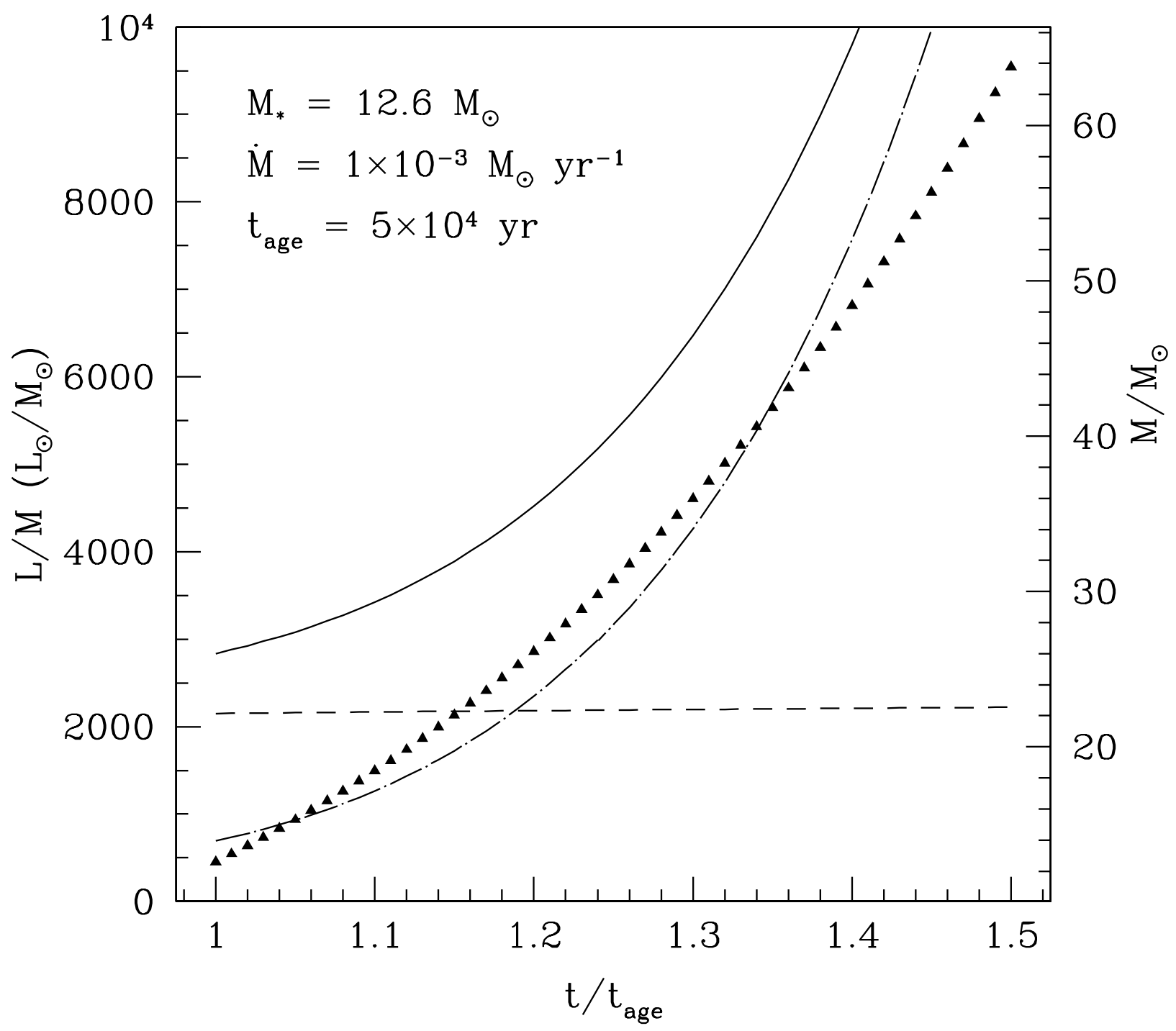

